\title{
Broad-based genomic sequencing in advanced non-small cell lung cancer in the dock
}

\author{
Caterina Fumagalli ${ }^{1}$, Elena Guerini-Rocco ${ }^{1,2}$, Massimo Barberis ${ }^{1}$ \\ ${ }^{1}$ Clinical Unit of Histopathology and Molecular Diagnostics, European Institute of Oncology, IRCCS, Milano, Italy; ${ }^{2}$ Department of Oncology and \\ Hemato-Oncology, University of Milano, Milano, Italy \\ Correspondence to: Massimo Barberis. Division of Pathology, European Institute of Oncology, IRCCS, Via Giuseppe Ripamonti 435, 20141 Milan, Italy. \\ Email: Massimo.Barberis@ieo.it. \\ Provenance: This is an invited article commissioned by the Section Editor Hengrui Liang (Department of Thoracic Surgery, Guangzhou Medical \\ University, Guangzhou, China). \\ Comment on: Presley CJ, Tang D, Soulos PR, et al. Association of Broad-Based Genomic Sequencing With Survival Among Patients With Advanced \\ Non-Small Cell Lung Cancer in the Community Oncology Setting. JAMA 2018;320:469-77.
}

Submitted Apr 09, 2019. Accepted for publication Apr 28, 2019.

doi: $10.21037 /$ tlcr.2019.04.16

View this article at: http://dx.doi.org/10.21037/tlcr.2019.04.16

The recently published study of Presley and colleagues (1) aimed to ascertain if there is an association between broadbased genomic sequencing (defined as multigene NextGeneration Sequencing panels of 30 or more genes) and better survival in patients with advanced non-small cell lung cancer (NSCLC). For this purpose, the authors retrospectively analyzed data obtained from medical records of a large cohort of patients $(n=5,688)$ with NSCLC from January 2011 to July 2016, treated in 191 community oncology practices across the United States. Overall, broadbased genomic sequencing was performed for 875 patients (15.4\%) and routine genomic testing (testing of EGFR/ $A L K$ only) for 4,813 patients (84.6\%). These two groups were compared for two parameters: the 12 -month mortality using an instrumental variable approach and the overall survival with a propensity score-matched survival analysis. According to these analyses, the authors found no significant association between broad-based genomic sequencing and 12 -month mortality [predicted probability of death: $41.1 \%$ for broad-based genomic sequencing vs. $44.4 \%$ for routine testing; difference $-3.6 \%$, $(95 \% \mathrm{CI},-18.4 \%$ to $11.1 \%)]$ or overall deaths [ $42.0 \%$ vs. $45.1 \%$; hazard ratio, 0.92 (95\% CI, 0.73-1.11)], despite the unadjusted KaplanMeier survival analysis showing better survival with broad genomic sequencing [hazard ratio 0.69 (95\% CI, 0.62-0.77); $\log$-rank $\mathrm{P}<0.001]$. The authors concluded that the use of broad-based genomic sequencing for advanced NSCLC in the community setting is not independently associated with a survival advantage. The study conducted by Presley et al. (1) presented interesting data about the use of broad genomic approach as compared to routine genomic testing across different community oncology practices and among a large cohort of patients with NSCLC. However, some considerations are needed regarding the usefulness of multigene approach and the potential clinical benefit of targeted treatment.

First, as the authors reported, at the time of the study routine testing for $E G F R / A L K$ only was considered the standard-of-care for patients with advanced NSCLC. However, the recently updated guidelines regarding the molecular testing in patients with advanced lung cancer, recommended a wider panel of genes to be tested in order to drive personalized therapy $(2,3)$ including $R O S 1$ and $B R A F$ evaluations as stand-alone tests and RET, ERBB2 (HER2), KRAS, and MET analyses as part of larger panels (2). Moreover, the number of biomarkers to be tested may rise rapidly as data are emerging about the oncogenic role of additional molecular aberrations that could be efficacy targeted by new drugs (e.g., NTRK1/2/3 fusion genes or NF1 mutations) (4,5). In our experience, using a multi-gene panel we could identify an overall rate of $32.6-39.8 \%$ of patients with advanced NSCLC harboring alterations targetable by therapies in a clinical setting or clinical trials $(6,7)$. This prevalence is also confirmed by the 
study of Presley (1) that reported $18 \%$ and $3.7 \%$ of EGFRmutated and $A L K$-rearranged cases respectively and identified additional alterations in ROS1, MET, BRAF, ERBB2, NTRK13 , and $R E T$ in at least 125 patients (14.3\%) using broad-based genomic sequencing. However, only a small number of these patients received a broad-based genomic sequencing directed targeted treatment $(n=36)$, leading to the conclusion that this broad sequencing approach may not currently improve survival (1). Undoubtedly, no survival advantage could be expected if the alteration is identified but the patient is not actively treated. This observation is valid regardless of the type of target treatment adopted, still fitting for EGFR or $A L K$ target therapies $(8,9)$. Therefore, the clinical benefit could be achieved only if the broad-based genomic sequencing is combined with a broad targeted drug availability.

Second, a multi-gene approach may add and not decrease information to data obtained from single-gene routine testing only. As reported by Presley (1), there was a high concordance (98-99.1\%) between 399 EGFR and 330 ALK tests performed in patients who received both broad-based genomic sequencing testing and single-gene testing. Beside, upfront multi-gene panels could be useful in terms of turnaround time, cost-effectiveness and tissue management $(10,11)$. Nowadays, Next Generation Sequencing (NGS) panels require a small quantity of input DNA and allow to simultaneously evaluate a large number of genes and different type of alterations, including single nucleotide variants, insertion or deletions, copy number variation, and fusion genes (12). On the other hand, serial testing of single genes may expand turnaround time and tissue requirement. This is especially true for advanced NSCLC-testing, where the samples are often endobronchial ultrasound biopsies, lung biopsies or cytological samples with limited amount of material (13). Moreover, a multi-gene panel approach could be useful not only at diagnosis but also in the monitoring of therapy response for the identification of acquired resistance mechanisms. Indeed, acquired resistance may be related not only to "on-target" mechanisms (e.g., secondary acquired T790M mutation in EGFR) but also to "off-target" mechanisms including bypass or downstream signaling pathway activation (e.g., MET alterations in EGFR-mutated NSCLC treated with EGFR inhibitors) (14).

Third, a multi-gene comprehensive analysis could be useful for clinical decision making not only about specific targeted therapy but also about different therapy regimens, including immunotherapy. In the last years, PD-1/PD-
L1 inhibitors have been rapidly emerging as a therapeutic option for patients with advanced NSCLC (15-17). In the study of Presley et al. (1) there was a significant association between the selection of immunotherapy as $1^{\text {st }}$ or $2^{\text {nd }}$ line treatment and the receipt of broad-based genomic sequencing $(\mathrm{P}<0.001)$. Even if these data were not discussed and the molecular profile of patients receiving immunotherapy was not reported, immunotherapy within the first $4^{\text {th }}$ lines of treatment was significantly associated with improved survival [adjusted hazard ratio 0.41 (95\% CI, 0.36-0.47), $\mathrm{P}<0.001]$. Tumors with ROS1 or $A L K$ rearrangement or $E G F R$ mutation frequently showed a high expression of PD-L1 $(18,19)$; however, the presence of these driver alterations has been recently associated with low response to PD-1/PD-L1 inhibitors (20). Prospective studies are thus warranted to evaluate the efficacy of immunotherapy in such patients. However, the exclusion of driver mutations using a broad-based sequencing approach may represent another important information provided to the clinicians to drive therapy decision. Moreover, although is still highly debated, tumor mutational burden may become an additional predictive biomarker for immunotherapy (21). In this scenario, only large (>1 Mb tumor coding genome covered) multi-gene NGS panels could be adopted (22).

In conclusion, the study conducted by Presley and colleagues provides important insights into the prevalence of broad-based genomic sequencing in the community oncology setting. The authors concluded that this sequencing approach might not currently offer a survival advantage in patients with NSCLC. However, assessing at the same time different individual diagnostic and prognostic risk factors as well as druggable alterations represents the main goal of the precision medicine (23). Indeed, the broad-based genomic sequencing may reveal its clinical utility only into the context of a multidisciplinary team setting, where clinical requests and molecular results could be joined and discussed to improve the therapeutic opportunity for each patient (24).

\section{Acknowledgments}

None.

\section{Footnote}

Conflicts of Interest: The authors have no conflicts of interest to declare. 


\section{References}

1. Presley CJ, Tang D, Soulos PR, et al. Association of Broad-Based Genomic Sequencing With Survival Among Patients With Advanced Non-Small Cell Lung Cancer in the Community Oncology Setting. JAMA 2018;320:469-77.

2. Kalemkerian GP, Narula N, Kennedy EB. Molecular Testing Guideline for the Selection of Lung Cancer Patients for Treatment With Targeted Tyrosine Kinase Inhibitors: American Society of Clinical Oncology Endorsement Summary of the College of American Pathologists/International Association for the Study of Lung Cancer/Association for Molecular Pathology Clinical Practice Guideline Update. J Oncol Pract 2018;14:323-7.

3. Lindeman NI, Cagle PT, Aisner DL, et al. Updated Molecular Testing Guideline for the Selection of Lung Cancer Patients for Treatment With Targeted Tyrosine Kinase Inhibitors: Guideline From the College of American Pathologists, the International Association for the Study of Lung Cancer, and the Association for Molecular Pathology. J Thorac Oncol 2018;13:323-58.

4. Halliday PR, Blakely CM, Bivona TG. Emerging Targeted Therapies for the Treatment of Non-small Cell Lung Cancer. Curr Oncol Rep 2019;21:21.

5. Drilon A, Laetsch TW, Kummar S, et al. Efficacy of Larotrectinib in TRK Fusion-Positive Cancers in Adults and Children. N Engl J Med 2018;378:731-9.

6. Fumagalli C, Vacirca D, Rappa A, et al. The long tail of molecular alterations in non-small cell lung cancer: a single-institution experience of next-generation sequencing in clinical molecular diagnostics. J Clin Pathol 2018;71:767-73.

7. Fumagalli C, Catania C, Ranghiero A, et al. Molecular Profile of Advanced Non-Small Cell Lung Cancers in Octogenarians: The Door to Precision Medicine in Elderly Patients. J Clin Med 2019;8. doi: 10.3390/jcm8010112.

8. Kris MG, Johnson BE, Berry LD, et al. Using multiplexed assays of oncogenic drivers in lung cancers to select targeted drugs. JAMA 2014;311:1998-2006.

9. Bunn PA Jr, Aisner DL. Broad-Based Molecular Testing for Lung Cancer: Precisely the Time for Precision. JAMA 2018;320:445-6.

10. Pedrizet K, Stockley T, Tsao MS, et al. Upfront next generation sequencing in NSCLC: A publicly funded perspective. J Clin Oncol 2018;36:12062.

11. Pennel NA, Mutebi A, Zhou ZY, et al. Economic impact of next generation sequencing vs sequential single- gene testing modalities to detect genomic alterations in metastatic non-small cell lung cancer using a decision analytic model. J Clin Oncol 2018;36:9031.

12. Suh JH, Johnson A, Albacker L, et al. Comprehensive Genomic Profiling Facilitates Implementation of the National Comprehensive Cancer Network Guidelines for Lung Cancer Biomarker Testing and Identifies Patients Who May Benefit From Enrollment in MechanismDriven Clinical Trials. Oncologist 2016;21:684-91.

13. Yu TM, Morrison C, Gold EJ, et al. Multiple Biomarker Testing Tissue Consumption and Completion Rates With Single-gene Tests and Investigational Use of Oncomine Dx Target Test for Advanced Non-Small-cell Lung Cancer: A Single-center Analysis. Clin Lung Cancer 2019;20:20-29.e8.

14. Rotow J, Bivona TG. Understanding and targeting resistance mechanisms in NSCLC. Nat Rev Cancer 2017;17:637-58.

15. Borghaei H, Paz-Ares L, Horn L, et al. Nivolumab versus docetaxel in advanced nonsquamous non-small-cell lung cancer. N Engl J Med 2015;373:1627-39.

16. Herbst RS, Baas P, Kim DW, et al. Pembrolizumab versus docetaxel for previously treated, PD-L1-positive, advanced non-small-cell lung cancer (KEYNOTE-010): a randomised controlled trial. Lancet 2016;387:1540-50.

17. Rittmeyer A, Barlesi F, Waterkamp D, et al. Atezolizumab versus docetaxel in patients with previously treated non-small-cell lung cancer (OAK): a phase 3, openlabel, multicentre randomised controlled trial. Lancet 2017;389:255-65.

18. Yoneshima Y, Ijichi K, Anai S, et al. PD-L1 expression in lung adenocarcinoma harboring EGFR mutations or ALK rearrangements. Lung Cancer 2018;118:36-40.

19. Lee J, Park CK, Yoon HK, et al. PD-L1 expression in ROS1-rearranged non-small cell lung cancer: A study using simultaneous genotypic screening of EGFR, ALK, and ROS1. Thorac Cancer 2019;10:103-10.

20. Gainor JF, Shaw AT, Sequist LV, et al. EGFR Mutations and ALK Rearrangements Are Associated with Low Response Rates to PD-1 Pathway Blockade in Non-Small Cell Lung Cancer: A Retrospective Analysis. Clin Cancer Res 2016;22:4585-93.

21. Hellmann MD, Ciuleanu TE, Pluzanski A, et al. Nivolumab plus ipilimumab in lung cancer with a high tumor mutational burden. N Engl J Med 2018;378:2093-104.

22. Büttner R, Longshore JW, López-Ríos F, et al. Implementing TMB measurement in clinical practice: considerations on assay requirements. ESMO Open 
2019;4:e000442. doi: 10.1136/esmoopen-2018-000442.

23. Matchett KB, Lynam-Lennon L, Watson W, et al. Advances in Precision Medicine: Tailoring Individualized Therapies. Cancers (Basel) 2017;9. doi: 10.3390/ cancers9110146.

Cite this article as: Fumagalli C, Guerini-Rocco E, Barberis M. Broad-based genomic sequencing in advanced non-small cell lung cancer in the dock. Transl Lung Cancer Res 2019;8(Suppl 4):S360-S363. doi: 10.21037/tlcr.2019.04.16
24. Rolfo C, Manca P, Salgado R, et al. Multidisciplinary molecular tumour board: a tool to improve clinical practice and selection accrual for clinical trials in patients with cancer. ESMO Open 2018;3:e000398. 